\title{
Potencial Agroindustrial del Epicarpio de Maracuyá como Ingrediente Alimenticio Activo
}

\author{
Junior B. Molina-Hernández, Hugo A. Martínez-Correa, Margarita M. Andrade-Mahecha \\ Universidad Nacional de Colombia, Sede Palmira, Dpto. de Ingeniería, Cr 32 \# 12-00, Palmira, Colombia. \\ (e-mail: jbmolinah@unal.edu.co; hamartinezco@unal.edu.co; mmandradem@unal.edu.co)
}

Recibido Ago. 2, 2018; Aceptado Oct. 18, 2018; Versión final Nov. 16, 2018, Publicado Abr. 2019

\begin{abstract}
Resumen
Se evaluaron las propiedades fisicoquímicas, tecnológicas, microbiológicas y antioxidantes de epicarpio de maracuyá (EM, Passiflora edulis f. flavicarpa). Los resultados mostraron un alto contenido de fibra dietaría tanto soluble como insoluble $(26,62$ y $44,84 \mathrm{~g} / 100 \mathrm{~g}$ b.s. respectivamente) y alto contenido de proteína $(11,87 \mathrm{~g} / 100 \mathrm{~g}$ b.s.). Con el fin de evaluar el potencial de uso de epicarpio de maracuyá como insumo para enriquecer productos alimenticios, se determinó la capacidad emulsionante (97\%), capacidad de hinchamiento (28.63 g/g b.s.), capacidad de retención de aceite $(4,06 \mathrm{~g} / \mathrm{g}$ b.s.), solubilidad $(0,71 \mathrm{~g} / \mathrm{g}$ b.s.) y el contenido de carotenoides totales $(3,86 \mathrm{mg} \beta$-carotenos $/ 100 \mathrm{~g})$. Adicionalmente, para la determinación de actividad antioxidante se utilizaron dos técnicas (DPPH y ABTS) con valores de actividad antioxidante del $33 \%\left(\mathrm{IC}_{50} \geq 250 \mu \mathrm{g} / \mathrm{ml}\right)$ y $2,09 \mu \mathrm{m} / \mathrm{g}$ b.s. de $\mathrm{EM}$ respectivamente. Los resultados de este estudio evidenciaron el potencial de uso del epicarpio de maracuyá como ingrediente funcional en la industria alimentaria.
\end{abstract}

Palabras clave: epicarpio de maracuyá; fibra dietaría total; capacidad antioxidante, ingredientes funcionales

\section{Agroindustrial Potential of Passion Fruit Epicarp as Active Food Ingredient}

\begin{abstract}
The physicochemical, technological, microbiological and antioxidant properties of the passion fruit epicarp (EM, Passiflora edulis f.flavicarpa) were evaluated. The results showed a high dietary fiber content both soluble and insoluble (26.62 and $44.84 \mathrm{~g} / 100 \mathrm{~g}$ d.b. respectively) and high protein content $(11.87 \mathrm{~g} / 100 \mathrm{~g}$ b.s.). In order to evaluate the potential use of passion fruit epicarp as an input to enrich food products, the emulsifying capacity (97\%), swelling capacity ( $28.63 \mathrm{~g} / \mathrm{g}$ d.b.), oil holding capacity (4.06 g / g d.b.), solubility $(0.71 \mathrm{~g} / \mathrm{g}$ d.b.) and the content of total carotenoids $(3.86 \mathrm{mg} \beta$-carotenes $/ 100 \mathrm{~g})$ were determined. Additionally, two techniques (DPPH and ABTS) with values of antioxidant activity of $33 \%$ (IC50 $\geq 250 \mu \mathrm{g} /$ $\mathrm{ml}$ ) and $2.09 \mu \mathrm{m} / \mathrm{g}$ d.b. were used to determine antioxidant activity. of EM respectively. The results of this study showed the potential use of the passion fruit epicarp as a functional ingredient in the food industry.
\end{abstract}




\section{INTRODUCCIÓN}

La producción de frutas tropicales alcanzó 85 millones de toneladas en 2014, lo cual representa un aumento de 38 millones de toneladas a partir de 1998 (FAO, 2014). Colombia es el segundo país productor de frutas tropicales en Latinoamérica debido en gran parte, a su ubicación geográfica y a la variedad de frutas disponibles, que exhiben excelentes cualidades sensoriales, nutricionales y / o nutracéuticas. Entre las frutas tropicales con gran importancia comercial se encuentra el maracuyá (Passiflora edulis) más conocido como el fruto de la pasión, el cual pertenece a la familia Passifloraceae. En 2011, la superficie dedicada al cultivo de maracuyá en Colombia fue de 5321 hectáreas, con una producción de $79.458 \mathrm{t}$, y un rendimiento promedio de 14,9 t/ Ha (López-Vargas et al., 2013). En 2013, el Valle del Cauca alcanzó una producción aproximada de 510.000 toneladas de frutas tropicales, de las cuales el 3,72\% correspondieron a la producción de maracuyá (Agronet, 2013). Este fruto se consume en fresco para producir jugo (alrededor del $40 \%$ del fruto corresponde a pulpa) o se comercializa como un importante componente de bebidas a base de frutas tropicales, debido a sus características nutritivas, coloración amarillo-naranja, sabor ácido y aroma exótico (Talcott et al., 2003).

Existen pocos estudios direccionados a explorar el potencial de residuos de maracuyá, los cuales son ricos en fibra. Algunos estudios han analizado la composición química y las propiedades antioxidantes de residuos de maracuyá como fuentes potenciales de fibra dietaría y polifenoles (López-Vargas et al., 2013; Hernández-Santos et al., 2015). Las fibras presentes en residuos de fruta, también pueden contener apreciables cantidades de pigmentos, antioxidantes $u$ otras sustancias con efectos positivos para la salud, entre los cuales se destaca, reducción del riesgo de padecer enfermedades cardiovasculares, mejoramiento de la función gastrointestinal y digestión de los lípidos contribuyendo así al control de peso y también a reducir el riesgo de desarrollar algunos tipos de cáncer y enfermedades en pacientes inmunodeprimidas (Marques et al., 2016).

Hoy en día, una gran variedad de alimentos, como los productos cárnicos (Fernández-López et al., 2008; Sánchez-Zapata et al., 2011), productos de panificación (Martínez-Cervera et al., 2011) y productos lácteos, se han enriquecido con fibra. La fibra presente en residuos de maracuyá se puede incorporar en productos alimenticios como agentes texturales, no calóricos para la sustitución parcial de grasa, con el fin de garantizar la retención de agua, aceite y la estabilidad de las emulsiones (Elleuch et al., 2011; López-Vargas et al., 2014). En este sentido, el aprovechamiento del epicarpio de maracuyá aumentaría la competitividad en la cadena de frutas y hortalizas en Colombia, puntualmente en el eslabón de las pasifloras, ya que el aprovechamiento del fruto seria casi en un $80 \%$, de igual forma podría motivar el desarrollo de nuevos productos o la reformulación de productos existentes, ya sea como materia prima o como sustituto de ingredientes para el desarrollo de alimentos funcionales. El objetivo del presente trabajo fue determinar las propiedades fisicoquímicas, tecnológicas, microbiológicas y antioxidantes presentes en el epicarpio de maracuyá, al igual que su potencial uso como fuente de fibra dietaría en alimentos de bajo contenido calórico.

\section{MATERIALES Y MÉTODOS}

Este estudio se desarrolló en la cuidad de Palmira (Valle del Cauca, Colombia) ubicada a $1050 \mathrm{msnm}$. Se describe la materia prima, el proceso de obtención de epicarpio de maracuyá deshidratado, se detallan los métodos usados para determinar la composición proximal, las propiedades tecnológicas, el contenido de carotenoides totales y la actividad antioxidante. Finalmente se detallan los análisis microbiológicos, el análisis de metales contaminantes, y se describe el análisis estadístico y diseño experimental utilizado.

\section{Materia prima}

$20 \mathrm{~kg}$ de epicarpio de maracuyá (Passiflora edulis $f$. flavicarpa) de color amarillo homogéneo en la superficie, sin cortes visibles en el exterior ni signos descomposición, fueron adquiridos como residuo del procesamiento y obtención de pulpa de maracuyá, cultivada en el municipio de Cerrito (Valle del Cauca) a $1000 \mathrm{msnm}$. Inmediatamente recolectados los residuos (epicarpio) se sumergieron en una solución de hipoclorito de sodio (100 ppm) durante 15 minutos. Posteriormente, se almacenaron a temperatura de refrigeración $\left(5^{\circ} \mathrm{C}\right)$ hasta su procesamiento.

\section{Proceso de obtención de epicarpio de maracuyá deshidratado}

Para la obtención de epicarpio de maracuyá (EM) se siguieron las condiciones reportadas por Espírito Santo et al. (2012) y Crizel et al. (2013) con algunas modificaciones. El epicarpio de maracuyá $(93,1 \%$ de humedad) se cortó en trozos (aproximadamente $2 \mathrm{~cm}^{2}$ ) y se sumergió en una solución de ácido cítrico 
durante 10 minutos. Posteriormente, el epicarpio se sometió a secado en un horno (IKA L. S., Alemania) con flujo de aire a $45^{\circ} \mathrm{C} / 18 \mathrm{~h}$ y humedad relativa de $39 \%$. El epicarpio seco $(7,56 \%$ de humedad) se sometió a molienda (Dida Control, Colombia) y el tamaño de partícula se estandarizó usando juego de tamices (Tyler) compuesto por malla $30(600 \mu \mathrm{m}), 60(250 \mu \mathrm{m}), 80(180 \mu \mathrm{m})$ y fondo. Con el fin de cumplir con los requerimientos establecidos por la NTC 267 (ICONTEC,2007) en relación al tamaño de partícula $\leq$ a 210 $\mu \mathrm{m}$, se utilizó el material que atravesó el tamiz con malla 80 (180 $\mu \mathrm{m}$ de tamaño). El material obtenido con tamaño de partícula inferior a $180 \mu \mathrm{m}$ correspondió al 42,7\% del material seco total, obteniendo un rendimiento del $7 \%$ en relación al material fresco. El epicarpio de maracuyá deshidratado molido (EM) se almacenó en un frasco ámbar previamente esterilizado y se mantuvo bajo refrigeración a $5^{\circ} \mathrm{C}$ hasta su uso.

\section{Composición proximal}

El contenido de humedad se determinó por triplicado empleando el método gravimétrico de secado en estufa (DIESI, D24OFU, Medellín), de acuerdo a la AOAC 925.09 (2000). Para la determinación del contenido de cenizas, las muestras fueron sometidas a incineración a $550{ }^{\circ} \mathrm{C}$ durante $6 \mathrm{~h}$ en una mufla (fischer scientific, 550 - 58), según método AOAC 923.03. (2005). El contenido total de fibra dietario, soluble e insoluble, fue determinado por el método AOAC 991.43 (1990). El contenido de proteína se determinó por triplicado con base al contenido de nitrógeno, mediante el método de Kjeldahl, empleando una unidad de digestión BUCHI, 350 (Suiza). Para la determinación del \% de grasa en el epicarpio de maracuyá se utilizó el método Bligh \& Dyer (1959).

\section{Propiedades fisicoquímicos}

La actividad de agua se determinó por triplicado empleando el equipo de actividad de agua (AquaLab 4te, USA), según AOAC 978.19 (2000). La acidez se determinó por triplicado expresándola como el porcentaje de ácido predominante (ácido galacturónico), con base al método descrito por la AOAC 942.15 (1990). Para estimar el pH se siguió el método AOAC 981.12. (1990), el cual consistió en tomar $5 \mathrm{~g}$ de muestra (tamaño de partícula: 180 - $250 \mu \mathrm{m}$ ) y diluirla en $50 \mathrm{~mL}$ de agua destilada. Posteriormente se procedió a la lectura empleando un potenciómetro (Metrohm, 744, USA) debidamente calibrado.

La medición de coordenadas CIELab en el epicarpio de maracuyá se realizó por triplicado en un colorímetro triestímulo (Chroma Meter-CR-400, Konica Minolta, USA) calibrado con un azulejo blanco estándar, cuya tabla de calibración fue $\mathrm{Y}=89.5, \mathrm{x}=0.3166$ y y=0.3347. Los parámetros que se usaron en la determinación fueron iluminante D65 y ángulo del observador $2^{\circ}$. Los parámetros $\mathrm{C} \mathrm{y}{ }^{\circ} \mathrm{H}$ se determinaron mediante las Ec. 1 y 2 respectivamente (Crizel et al., 2013).

$$
\begin{gathered}
C=\sqrt{a^{* 2}}+\sqrt{b^{* 2}} \\
{ }^{\circ} \mathrm{H}=180+\operatorname{arct} \frac{\mathrm{b}^{*}}{\mathrm{a}^{*}}
\end{gathered}
$$

Donde, C: saturación del color (croma); a*: coloración rojiza (+) o verde (-); b*: coloración amarilla (+) o azul $(-)$; ${ }^{\circ} \mathrm{H}$ : ángulo de tono; L*: luminosidad.

El Poder de hinchamiento y solubilidad se determinaron a diferentes temperaturas $\left(55,65,75,85\right.$ y $\left.95^{\circ} \mathrm{C}\right)$ mediante el método reportado por $\mathrm{Yu}$ et al. (2012) con algunas modificaciones. 0,16 g de epicarpio de maracuyá deshidratado $(212 \mu \mathrm{m})$ en base seca se incorporaron a un tubo de $15 \mathrm{ml}$, el cual contenía previamente $10 \mathrm{~mL}$ de agua destilada. Los tubos se mantuvieron a $55^{\circ} \mathrm{C}$ (inicialmente) con agitación mediante un baño térmico Julabo, Shake Temp SW22 (Suiza), se centrifugaron en un equipo Centurion Scientific K241L (Reino Unido). El residuo retenido en el tubo y el sobrenadante se pesaron en una balanza analítica (kern y Sohn GmbH ABJ 220-4M, Suiza). El sobrenadante se colocó en una caja petri para ser sometida a secado en horno (DIESI, D240FU, Medellín) a $105^{\circ} \mathrm{C}$ por $2 \mathrm{~h}$. El poder de solubilidad (\% S.S.) e hinchamiento (P.H) se determinaron empleando las Ec.3 y 4

$$
\begin{aligned}
& \% \text { S.S. }=\frac{\text { masa sobrenadante seco }(\mathrm{g})}{\text { masa de la muestra }(\mathrm{g})} \times 100 \\
& \% \text { P.H. }=\frac{\text { masa de residuo humedo }(\mathrm{g})}{\text { masa de la muestra }(\mathrm{g})-\text { masa de sobrenadante seco }(\mathrm{g})} \times 100
\end{aligned}
$$


Para la determinación de Capacidad de retención de aceite se siguió el método descrito por Wani et al. (2013), el cual consistió en medir la absorción de aceite de $1 \mathrm{~g}$ de muestra de epicarpio de maracuyá (220 $\mu \mathrm{m})$. Las muestras se incorporaron en tubos falcón de $50 \mathrm{ml}$, se adicionaron $10 \mathrm{~mL}$ de aceite de girasol, se mezclaron durante $1 \mathrm{~min}$. y se sometieron a centrifugación. El aceite liberado durante la centrifugación se drenó y se determinó la capacidad de absorción de aceite expresándolo como g de aceite retenido por cada $\mathrm{g}$ de muestra de epicarpio de maracuyá deshidratado.

La Capacidad emulsionante y estabilidad de la emulsión se determinaron siguiendo el método descrito por Kaur y Singh (2005) con algunas modificaciones. El método consistió en pesar 5,25 g de muestra de epicarpio de maracuyá. Esta se mezcló con $100 \mathrm{~mL}$ de agua destilada y se homogenizo empleando ultraturrax (IKA, T25 Basic). Posteriormente, se adicionaron $50 \mathrm{~mL}$ de aceite en dos partes continuando con la homogenización. La emulsión se centrifugó y la actividad emulsificante se calculó dividiendo el volumen de la capa emulsionada en el volumen de emulsión antes de la centrifugación. La estabilidad de la emulsión se determinó usando las muestras preparadas para la actividad emulsificante, estas se calentaron durante 15 min a $85^{\circ} \mathrm{C}$ empleando baño térmico, se enfriaron a temperatura ambiente $\left(26 \pm 2^{\circ} \mathrm{C}\right)$ y se centrifugaron nuevamente. La estabilidad de la emulsión se expresó cómo \% de actividad emulsionante después del calentamiento.

\section{Contenido de carotenoides totales y actividad antioxidante}

La importancia de los carotenoides va más allá de las funciones biológicas como patrones de coloración en plantas, animales y protección del ataque de las proteasas en algunos invertebrados. Estos compuestos tienen propiedades biológicas y se consideran precursores de la vitamina $A$, también llamados provitamina A (Fernández-García et al., 2012). Por lo cual determinar su contenido en productos que se incluirán en la formación de alimentos es de relevante importancia. Los carotenos totales se cuantificaron por triplicado de acuerdo a la metodología reportada por Barrett et al (2001), con algunas modificaciones de Ordoñez Santos et al. (2014).

Extracción. Se pesaron $0,1 \mathrm{~g}$ de muestra, la cual se colocó en un tubo de ensayo, previamente cubierto con papel aluminio. Posteriormente se adicionaron $7 \mathrm{~mL}$ de la mezcla etanol-hexano (4:3 v/v) y se llevaron a baño térmico $\left(16^{\circ} \mathrm{C}\right)$ por $1 \mathrm{~h}$ con agitación mediante un shacker (Orbital mrC, TOS-4030f). Finalmente, se adicionó $1 \mathrm{~mL}$ de agua destilada y se agito nuevamente.

Cuantificación. La Absorbancia a una longitud de onda de $450 \mathrm{~nm}$ fue medida, en un espectrofotómetro (Jenway, 6320D, Inglaterra) previamente calibrado con hexano como blanco. Posteriormente, se calculó la concentración de carotenoides totales ( $\beta$-caroteno) empleando la ecuación de Lambert- Beer (Ec.5).

$$
\text { C.T. }\left[\frac{\mathrm{mg}}{100 \mathrm{~g}}\right]=\frac{A * V * 10^{3}}{E^{1 \%} * W}
$$

Donde, "A" es la absorbancia a $450 \mathrm{~nm}$, "v" es el volumen del extracto en mililitros, "w" es el peso de la muestra en gramos y E1\% es el coeficiente de extinción para $\beta$ caroteno $(2560 \mathrm{~cm}-\mathrm{I})$

Actividad antioxidante por método DPPH: siguiendo la metodología reportada por Mensor et al. (2001), utilizando el reactivo (1,1-difenil-2-picrilhidrozilo) y Etanol grado reactivo. Se procedió a preparar una solución a una concentración de $1 \mathrm{mg} / \mathrm{ml}$ a partir de la cual se elaboraron diferentes soluciones con concentraciones finales entre $25-250 \mu \mathrm{g} / \mathrm{ml}$. Posteriormente se preparó la solución de DPPH, tomando 3 $\mathrm{mg}$ de reactivo DPPH, los cuales se disolvieron en $15 \mathrm{ml}$ de solución extractora (Etanol grado reactivo), completando un total de $25 \mathrm{ml}$ de solución. Se tomaron alícuotas de $2,5 \mathrm{ml}$ de cada una de las concentraciones elaboradas y $1 \mathrm{ml}$ de solución de DPPH las cuales se llevaron a tubos de ensayo protegidos de la luz, por 30 minutos a temperatura ambiente. Se midió la absorbancia a $518 \mathrm{~nm}$ utilizando un espectrofotómetro (Thermo Scientific, Genesys UV VIS; U.S.A). El blanco se preparó para cada una de las concentraciones elaboradas, mezclando $1 \mathrm{ml}$ de etanol con $2,5 \mathrm{ml}$ de extracto, y el control negativo mediante la mezcla de $1 \mathrm{ml}$ de solución de $\mathrm{DPPH}$ y $2,5 \mathrm{ml}$ de etanol. Para la determinación del porcentaje de actividad antioxidante se utilizó la Ec. 6

$\%$ A.A. $=\frac{100-(\text { Absmuestra }- \text { Absblanco })}{\text { Abscontrol }} \times 100$

Método ABTS (2,2'-azino-bis (3-ethilbenzothiazolina-6- ácido sulfonico)): La determinación de capacidad antioxidante del extracto de EM en el radical ABTS + se llevó a cabo siguiendo la metodología reportada 
por, Larrauri (1997) con algunas modificaciones. El catión ABTS + fue producido por la reacción de oxidación entre $5 \mathrm{ml}$ de solución acuosa de ABTS (Sigma, Aldrich) $(7 \mathrm{mM}$ ) y $84 \mu \mathrm{l}$ de Persulfato de Potasio (Panreac, Aplichemm) (140 mM). La mezcla se almacenó a temperatura ambiente en espacio oscuro por 16 h. Posteriormente se diluyo en etanol hasta conseguir una absorbancia de 0,700 $\pm 0,050$ a $734 \mathrm{~nm}(1,091 \%$ $\mathrm{v} / \mathrm{v})$. Se prepararon mezclas de extracto de EM + ABTS: en un ambiente oscuro se transfirieron alícuotas de $30 \mu \mathrm{l}$ del extracto de cada dilución a tubos de ensayo y se completó con $3 \mathrm{ml}$ de solución ABTS+. Se realizaron lecturas a $734 \mathrm{~nm}$ después de 6 minutos de reacción, se utilizó alcohol etílico como blanco y para la calibración del espectrofotómetro. La curva estándar fue lineal $100 \mu \mathrm{M}$ y $2.000 \mu \mathrm{M}$ de trolox. La actividad antioxidante se expresó como $\mu \mathrm{m}$ de trolox/mg de extracto seco.

\section{Análisis microbiológicos}

Para dar cumplimento a los requerimientos microbiológicos establecidos en la NTC 267 (ICONTEC,2007) numeral 4.2.2. en harinas vegetales, muestras de epicarpio de maracuyá deshidratado fueron evaluados por un laboratorio externo acreditado para análisis microbiológicos con el fin determinar: Recuento total de Aerobios mesofilos, Recuento de Mohos y Levaduras, Recuento de Escherichia coli, Investigación de Salmonella en $25 \mathrm{~g}$, Recuento de Bacillus cereus y Recuento de Estafilococo Coagulasa positiva.

\section{Análisis de metales contaminantes}

Para dar cumplimento a los requerimientos establecidos en la NTC 267 (ICONTEC,2007) numeral 4.2.4. sobre metales contaminantes en harinas vegetales, muestras de epicarpio de maracuyá deshidratado fueron evaluados por un laboratorio externo acreditado con el fin de determinar: contenido de plomo y cadmio (mg/kg), para ello se utilizó el método ICP-OES (Plasma de acoplamiento inductivo espectrofotómetro de emisión óptico).

\section{Análisis estadístico}

Se realizó un análisis de varianza (ANOVA) y una prueba de Tukey, con un nivel de significación del 5\% utilizando el software SAS $9.4 \AA$ (EE. UU.) para comparar las diferencias entre medias de los diferentes análisis. Los experimentos y análisis que se realizaron se llevaron a cabo por triplicado y se informaron como la media \pm desviación estándar de los ensayos independientes.

\section{RESULTADOS Y DISCUSIÓN}

En la tabla 1 se presenta la composición proximal de epicarpio de maracuyá (EM). El contenido total de fibra dietaría (FDT) fue aproximadamente de $71,46 \mathrm{~g} / 100 \mathrm{~g}$ b.s. Según lo reportado por Larrauri (1999), productos con un contenido de fibra superior a $50 \mathrm{~g} / 100 \mathrm{~g}$ b.s. pueden ser considerados de alto contenido de fibra dietaría. Los valores de FDT fueron cercanos a los reportados por Martínez et al. (2012) para epicarpio de mango, piña y guayaba (70; 75.8 y $69.1 \mathrm{~g} / 100 \mathrm{~g}$ b.s. respectivamente). En el presente estudio, el contenido fibra del epicarpio de maracuyá, fue superior a lo reportado por Hernández-Santos et al. (2015), Macagnan et al. (2015) y Lima et al. (2016) para epicarpio de maracuyá (Passiflora edulis f. flavicarpa) $(57,93 \mathrm{~g} / 100 \mathrm{~g}$, $62,65 \mathrm{~g} / 100 \mathrm{~g}, 60,08 \mathrm{~g} / 100 \mathrm{~g}$ respectivamente). Así al comparar con otros tipos de residuos se evidencia que el epicarpio de maracuyá presenta un alto contenido de fibra. Chau y Huang, (2004) reportaron un contenido de fibra en semilla de maracuyá de 64,8 g /100g y Macagnan et al. (2015) para bagazo de naranja de $54.82 \mathrm{~g} / 100 \mathrm{~g}$. Estas diferencias entre residuos se pueden atribuir al tipo de cultivo, variedades y estado de maduración del fruto (Crizel et al., 2013). El contenido de fibra dietaría insoluble (FDI) y soluble (FDS) se reportan en la tabla 1. FDI fue superior a FDS (44,84 y $26,62 \mathrm{~g} / 100 \mathrm{~g}$ b.s. respectivamente). El contenido de FDI fue similar al reportado por Martínez et al. (2012) para epicarpio de mango y superior al reportado por Macagnan et al. (2015) y Lima et al. (2016) para epicarpio de maracuyá $(41,5 ; 43,43$ y 39.96 $\mathrm{g} / 100 \mathrm{~g}$ b.s respectivamente), y ligeramente inferior al reportado por López y Vargas et al. (2013) para albedo (52,34 g/100g b.s) y pulpa-semilla de maracuyá (Passiflora edulis $f$. flavicarpa). (48,25 g/100g b.s respectivamente). El contenido de FDI presente en epicarpio de maracuyá es un indicador del alto contenido de hemicelulosa, celulosa y lignina (López y Vargas et al., 2013; Martínez et al., 2012). Estos resultados son positivos teniendo en cuenta que el contenido de FDI puede ser utilizado con fines en la industria alimentaria, lo cual podría repercutir favorablemente en algunas funciones del sistema digestivo de los consumidores tales como incremento de la saciedad y volumen de las heces fecales.

El contenido de FDS fue superior al reportado por López y Vargas et al. (2013) para albedo de maracuyá $(19,45 \mathrm{~g} / 100 \mathrm{~g}$ b.s.), al reportado por Hernández y Santos et al. (2015) para epicarpio de maracuyá $(10,57$ g/100 g b.s.), López y Vargas et al. (2013) para pulpa y semilla de maracuyá (5,26 y 0,73 g/100g b.s respectivamente), y Martínez et al. (2012) para epicarpio de piña y guayaba (0,6 y 11,1 g/100 g b,s. respectivamente). Al comparar con lo reportado por Macagnan et al. (2016) se encontró un bajo contenido 
de FDS en algunas bebidas comparadas con EM: vino tinto $(2,29 \mathrm{~g} / 100 \mathrm{~g}$ b.s), leche de coco $(8,67 \mathrm{~g} / 100 \mathrm{~g}$ b.s.), jugo de naranja (0,95 g/100g b.s.), jugo de manzana (1,72 g/100g b.s.) y jugo de melocotón (2,19 $\mathrm{g} / 100 \mathrm{~g}$ b.s.). El alto contenido de FDS en epicarpio de maracuyá, indica que es una interesante alternativa para su incorporación como sustituto de grasa en la formulación de alimentos, debido a que poseen este tipo de fibras poseen una alta capacidad de retención de agua, aceite y capacidad emulsionante (Elleuch et al., 2011; López- Vargas et al., 2014). De igual forma es relevante su alto contenido de FDS en relación a bebidas alimenticias teniendo en cuenta que es un residuo del procesamiento de frutas, con un valor agregado tan alto como lo es la fuente de fibra dietaría. Por otro lado, estudios recientes han demostrado la estrecha relación de la fibra dietaría soluble presente en residuos de epicarpio de maracuyá y el beneficio en pacientes con inmunodeficiencia adquirida (VIH).

Estos pacientes presentan serios problemas de dislipemia, con lo cual se genera un aumento excesivo de colesterol en sangre, la fibra soluble permite reducir considerablemente estos niveles debido a las propiedades tecnológicas que presentan (Marques et al., 2016). Epicarpio de maracuyá deshidratado presento un bajo contenido de humedad (7,56 \%) similar a los valores reportados por Macagnan et al. (2015) para epicarpio de maracuyá (7,38\%) y Crizel et al. (2013) para epicarpio de mandarina (7,9 g/100g b.s) e inferior a lo reportado por Martínez et al. (2012) para epicarpio de mango, piña, guayaba, y maracuyá $(9,4 ; 9,3 ; 9,3$ y $9,3 \mathrm{~g} / 100 \mathrm{~g}$ b.s. respectivamente). Los valores de actividad de agua (aw) se reportan en la tabla 1 en encontrándose en el rango de 0,39 $\pm 0,02$. Este valor fue relativamente alto en comparación a lo reportado por López Vargas et al. (2013) para mesocarpio de maracuyá $(0,164)$ y Crizel et al. (2013) para epicarpio de mandarina (0.38). Los valores de $\mathrm{pH}$ y acidez fueron bajos (5,38 y 0,69\% respectivamente), los cuales fueron cercanos a lo reportado por López y Vargas et al. (2013) para mesocarpio de maracuyá $(4,36)$. Los valores de aw, humedad y $\mathrm{pH}$ (propiedades directamente relacionados con el deterioro de los alimentos) obtenidos para epicarpio de maracuyá en el presente estudio, indican el bajo riesgo de deterioro, que es causado principalmente por el crecimiento de microorganismos y el desarrollo de reacciones enzimáticas y no-enzimáticas, las cuales serían mínimas (López y Vargas et al., 2013), debido a que la degradación no ocurre aceleradamente en alimentos con baja actividad de agua (en un rango alrededor de 0,11-0,40 (Fernández- López 2008).

Tabla 1: Propiedades fisicoquímicas y contenido de carotenoides de epicarpio de maracuyá EM (g/100g b.s.).

\begin{tabular}{|c|c|}
\hline \multicolumn{2}{|c|}{ Epicarpio de maracuyá deshidratado } \\
\hline Parámetros evaluados & HEM \\
\hline \multicolumn{2}{|c|}{ Análisis Proximal } \\
\hline Humedad & $7,56 \pm 0.30$ \\
\hline Cenizas & $9,68 \pm 0.13$ \\
\hline FDT & $71,46 \pm 0.46$ \\
\hline FDS & $26,62 \pm 0.04$ \\
\hline FDI & $44,84 \pm 1.40$ \\
\hline Proteína & $11,87 \pm 0.08$ \\
\hline Grasa & $0,66 \pm 0.06$ \\
\hline \multicolumn{2}{|c|}{ Análisis Fisicoquímicos } \\
\hline Actividad de agua & $0,39 \pm 0.02$ \\
\hline Acidez (\%) & $0,69 \pm 0.02$ \\
\hline $\mathrm{Ph}$ & $5,38 \pm 0.04$ \\
\hline \multicolumn{2}{|c|}{ Parámetros de Color } \\
\hline $\mathrm{L}^{*}$ & $83,1 \pm 0.37$ \\
\hline$a^{*}$ & $-0,317 \pm 0.05$ \\
\hline$b^{*}$ & $19,43 \pm 0.05$ \\
\hline $\mathrm{C}^{*}$ & $19,43 \pm 0.05$ \\
\hline $\mathrm{H}^{*}$ & $122,7 \pm 0.0$ \\
\hline Carotenoides Totales (mg/100g) & $3,86 \pm 0.3$ \\
\hline
\end{tabular}


El contenido de proteína fue de $11,87 \mathrm{~g} / 100 \mathrm{~g}$ b.s., resultado muy superior a lo reportado por Crizel et al. (2013) para epicarpio de mandarina (1,81 g /100g b.s.), Martínez et al. (2012) para epicarpio de mango, piña, guayaba y maracuyá $(8,4 ; 4,8$ y $6,2 \mathrm{~g} / 100 \mathrm{~g}$ b.s. respectivamente), Macagnan et al. (2015) para pulpa de manzana (6.98 g/ $100 \mathrm{~g}$ b.s.), López y Vargas et al. (2013) para mesocarpio de maracuyá (0,39 g/100g b.s.) y Chau y Huang, (2004) para semilla de maracuyá ( $8.05 \mathrm{~g} / 100 \mathrm{~g}$ b.s.). El contenido de grasa fue bajo (0,66 $\pm 0.06 \mathrm{~g} / 100 \mathrm{~g}$ b.s), en comparación a lo reportados por otros autores, tales como, Crizel et al. (2013) para epicarpio de mandarina (1,81 g/100 g b.s.), Martínez et al. (2012) para epicarpio de mango, piña y guayaba $(5,9 ; 1,3$ y 2,4 g/100g b.s. respectivamente), y López y Vargas et al. (2013) para mesocarpio de maracuyá $(0,8 \mathrm{~g} / 100 \mathrm{~g}$ b.s.). En la misma dirección, Hernández y Santos et al. (2015) reportaron valores cercanos $(0,64 \mathrm{~g} / 100 \mathrm{~g}$ b.s) a los obtenidos para epicarpio de maracuyá (EM). El contenido de cenizas (9,68 $\mathrm{g} / 100 \mathrm{~g}$ b.s) para EM fue alto, al compararlo con lo reportado por otros autores para el mismo tipo de residuo: Hernández y Santos et al. (2015), López y Vargas et al. (2013), (6,44; 5 g b.s. respectivamente). El bajo contenido de grasa y alto contenido de proteína es un resultado favorable para las propiedades funcionales conferidas por las proteínas en las matrices alimentarias, y a su vez el bajo aporte calórico, el cual le confiere su potencial aplicación en alimentos. El alto contenido de cenizas podría ser una limitante teniendo en cuenta que el alto contenido de iones metálicos podría acelerar algunos procesos de oxidación en los componentes que hacen parte de la formulación del alimento (López y Vargas et al., 2013).

En la tabla 1 también se presentan los parámetros de color $\left(L^{*}, a^{*}, b^{*}, C^{*}\right.$ y $\left.H^{*}\right)$ para EM. El valor de luminosidad $\left(\mathrm{L}^{*}\right)$ fue de 83,17 . Para las coordenadas del rojo-verde $\left(\mathrm{a}^{*}\right)$ y azul-amarillo $\left(\mathrm{b}^{*}\right)$ fueron de $-0,317$ y 19,43 respectivamente. Por otro lado, el diferencial Croma $\left(\mathrm{C}^{*}\right)$ y el ángulo de tono $\left(\mathrm{H}^{*}\right)$ fueron de 19,43 y 122,70 respectivamente. Los resultados mencionados indican que EM presenta tonalidad entre el verde y amarillo. La incorporación de productos ricos en fibra en la elaboración de alimentos afecta considerablemente parámetros organolépticos como el color. Siendo entre los parámetros de calidad, los más importantes cuando son elaborados productos con alto contenido de fibra. Por lo tanto, uno de los mayores desafíos para la industria alimentaria, son los cambios de color y textura cuando se incorporan compuestos ricos en fibra en la formulación de un producto (Scott -Thomas, 2011).

\section{Propiedades Tecnológicas}

Los resultados sobre las propiedades funcionales presentadas en la tabla 2 evidencian que hubo diferencias significativas $(P \leq 0.05)$ entre las diferentes temperaturas para las propiedades sólidos solubles y poder de hinchamiento. La solubilidad aumentó a medida que lo hizo la temperatura, pasando de 0,275 g/100gb.s. $\left(55^{\circ} \mathrm{C}\right)$ a $0,392 \mathrm{~g} / 100 \mathrm{~g}$ b.s. $\left(65^{\circ} \mathrm{C}\right)$ y $0,484 \mathrm{~g} / 100 \mathrm{~g}$ b.s. $\left(75^{\circ} \mathrm{C}\right)$. Posteriormente se observó un aumento hasta valores de $0,715 \mathrm{~g} / 100 \mathrm{~g}$ secos a $95^{\circ} \mathrm{C}$. Resultados inferiores a lo reportado por Crizel et al. (2013) para epicarpio de mandarina $\left(2,3 \mathrm{~g} / 100 \mathrm{~g}\right.$ secos a $\left.105^{\circ} \mathrm{C}\right)$. Según estos autores por encima de $90^{\circ} \mathrm{C}$ la solubilidad tiende a disminuir debido a la deshidratación de las fibras. La alta solubilidad obtenida para el epicarpio de maracuyá posiblemente se debe a su alto contenido de fibra soluble (pectina y algunas hemicelulosas), las cuales contienen en su estructura grupos de sustitución tales como $\mathrm{COOH}$ o $\mathrm{SO}_{4-2}$ (carboxilos y sulfatos) que incrementan su solubilidad (Elleuch et al., 2011).

Tabla 2: Propiedades funcionales de epicarpio de maracuyá (HEM).

\begin{tabular}{|c|c|c|c|c|c|}
\hline Propiedades & $55^{\circ} \mathrm{C}$ & $65^{\circ} \mathrm{C}$ & $75^{\circ} \mathrm{C}$ & $85^{\circ} \mathrm{C}$ & $95^{\circ} \mathrm{C}$ \\
\hline $\begin{array}{c}\text { Solidos Solubles } \\
\text { (g/g secos) }\end{array}$ & $0,275 \pm 0.02^{\mathrm{cb}}$ & $0,392 \pm 0.03^{\mathrm{cb}}$ & $0,484 \pm 0.01^{\mathrm{c}}$ & $0,450 \pm 0.17^{\mathrm{b}}$ & $0,715 \pm 0.01^{\mathrm{a}}$ \\
\hline $\begin{array}{c}\text { Poder de } \\
\text { Hinchamiento } \\
\text { (g/g secos })\end{array}$ & $28,63 \pm 0.997^{\mathrm{a}}$ & $18,02 \pm 1.89^{\mathrm{b}}$ & $14,7 \pm 0.62^{\mathrm{c}}$ & $13,57 \pm 0.10^{\mathrm{c}}$ & $10,705 \pm 0.29^{\mathrm{d}}$ \\
\hline $\begin{array}{c}\text { Capacidad de retención de aceite } \\
\text { (g aceite/g epicarpio) }\end{array}$ & \multicolumn{2}{|c|}{ Capacidad emulsionante } & Estabilidad de la emulsión (\%) \\
\hline \multicolumn{2}{|c|}{$(\%)$} & \multicolumn{2}{|c|}{$100 \pm 0.6$} \\
\hline
\end{tabular}

La capacidad de hinchamiento es una propiedad que posee un material para retener agua cuando es sometido a una fuerza centrípeta externa. Generalmente, los polisacáridos presentes en las fibras dietarías son altamente hidrófilos, el agua es absorbida en los sitios polares de las fibras y dentro de los espacios vacíos (porosos) de la estructura molecular. La fibra dietaría participa en el aumento de esta propiedad, así 
la fibra insoluble aumenta la capacidad de hinchamiento debido a la alta porosidad que presentan y las fibras solubles tales como gomas y pectinas aumentan la viscosidad, ya que absorben agua y formar una masa gelatinosa (Mudgil y Barak, 2013). Los resultados del hinchamiento presentados por el epicarpio de maracuyá a diferentes temperaturas (tabla 2) hubo diferencias significativas entre muestras $(P \leq 0.05)$. Así el poder de hinchamiento disminuyó a medida que aumentó la temperatura, pasando de 28,63 g/100g b.s. $\left(55^{\circ} \mathrm{C}\right)$ a $10,72 \mathrm{~g} / 100 \mathrm{~g}$ b.s. $\left(95^{\circ} \mathrm{C}\right)$.

Resultados cercanos a los reportados por Crizel et al. (2013), Martínez et al. (2012) y López y Vargas et al. (2013) para epicarpio de mandarina, maracuyá y mesocarpio de maracuyá $(9,63 ; 13,05 ;$ y 13 g/100g secos respectivamente). En la figura 1 se puede observar la estructura de una partícula de EM seca (figura 1a) e hidratada (figura 1b), donde se aprecian estructuras lineales, posiblemente relacionadas con la presencia de fibra dietaría y un aumento del $200 \%$ en tamaño de la partícula hidratada en relación a la partícula seca, atribuido a su alta capacidad de hinchamiento y retención de agua. Epicarpio de maracuyá deshidratado con alta capacidad de hinchamiento pueden ser usado como ingrediente para alimentos funcionales con el fin de reducir calorías, sinéresis y modificar la textura y viscosidad del producto final. Estudios realizados en epicarpio de limón demostraron que, al disminuir el tamaño de partícula, aumenta esta propiedad, lo cual indica que el tamaño influye notablemente en este parámetro (Lario et al., 2004).

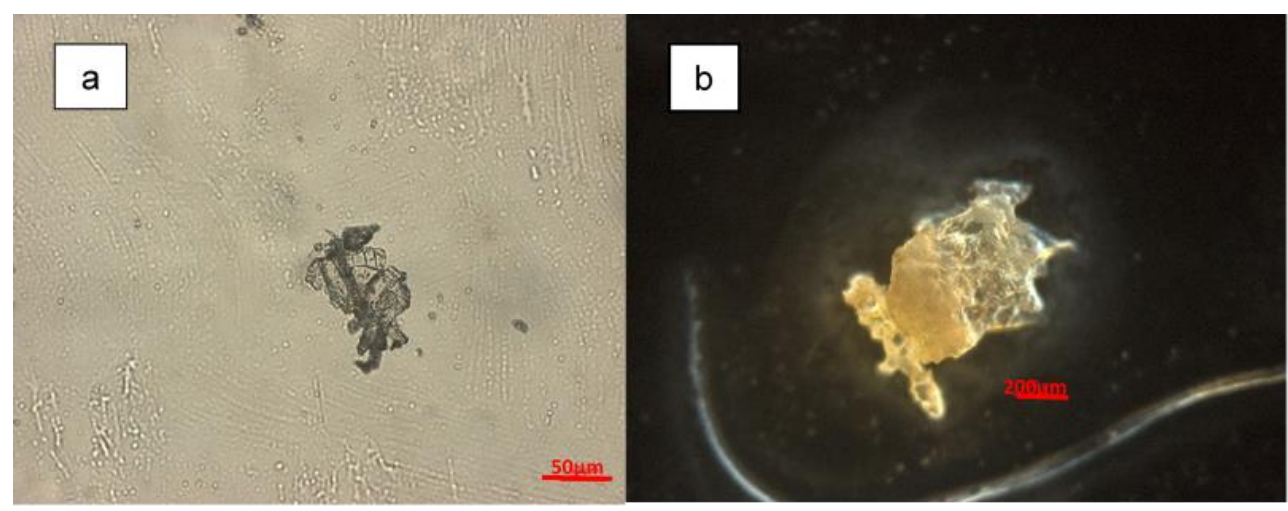

Fig. 1: Partícula de EM. a. Seca; b. Hidratada

Capacidad de retención de aceite y capacidad emulsionante también se muestran en la tabla 2. Epicarpio de maracuyá mostró valores altos (4,06 $\mathrm{g} / \mathrm{g}$ epicarpio) en relación a lo reportado por otros autores, tales como Crizel at al. (2013) para epicarpio de mandarina, López y Vargas et al. (2013) para mesocarpio de maracuyá y Martínez et al. (2012) para epicarpio de mandarina, mango, piña y guayaba (3,63; 2,4; 1,6; 0,7 y $0,7 \mathrm{~g}$ de aceite/ $\mathrm{g}$ de epicarpio b.s. respectivamente) para la propiedad capacidad de retención de aceite. La capacidad emulsionante del epicarpio de maracuyá fue del $94 \%$, siendo estable al 100\% después de ser sometido a calentamiento. Estos resultados están directamente relacionados con el alto contenido de fibra soluble (pectina) y proteína presente en EM (tabla 1), lo cual le confiere una alta capacidad de retener aceite, pero también una alta capacidad emulsionante (Martínez el al., 2012).

\section{Capacidad Antioxidante y carotenoides totales}

La importancia de los carotenoides va más allá de las funciones biológicas como patrones de coloración en plantas y animales, posee compuestos que tienen propiedades antioxidantes y se consideran precursores de la vitamina A, también llamados provitamina A. Por lo cual determinar su contenido en productos que se incluirán en la formación de alimentos es de alta importancia. En el presente estudio, el contenido de carotenoides en EM fue de 3,85 \pm 0,3 expresado como $\mathrm{mg} \beta$-carotenos/ $100 \mathrm{~g}$. Este resultado fue cercano al reportado por otros autores, tales como Hernández y Santos et al. (2015) para epicarpio de maracuyá (4,5 mg $\beta$-carotenos $/ 100 \mathrm{~g}$ ) y bajo en relación a lo reportado por Da Costa et al. (2013) para pulpa de maracuyá (8,3 mg $\beta$-carotenos/ 100g). El epicarpio es un residuo y el contenido de carotenoides es cercano al encontrado en un jugo de fruta. Por lo tanto, el epicarpio de maracuyá podría ser considerado como un ingrediente funcional. La reacción del radical libre 1,1-difenil-2-picrilhidroxilo (DPPH) con extracto de epicarpio de maracuyá (EM) y extractos patrones (ácido gálico, ascórbico y catequina) usados como antioxidantes de referencia, permitieron inhibir y estabilizar el radical libre, promoviendo cambios de color púrpura a blanco, lo cual conllevó a una disminución de la absorbancia. Para este método la actividad antioxidante se presentó en términos de $\mathrm{IC}_{50}$ (concentración de extracto necesaria para alcanzar $50 \%$ de actividad antioxidante). En la figura 2 se reporta el porcentaje de actividad antioxidante, la cual corresponde a la cantidad de radical DPPH neutralizado por el extracto a una determinada concentración de extracto. 


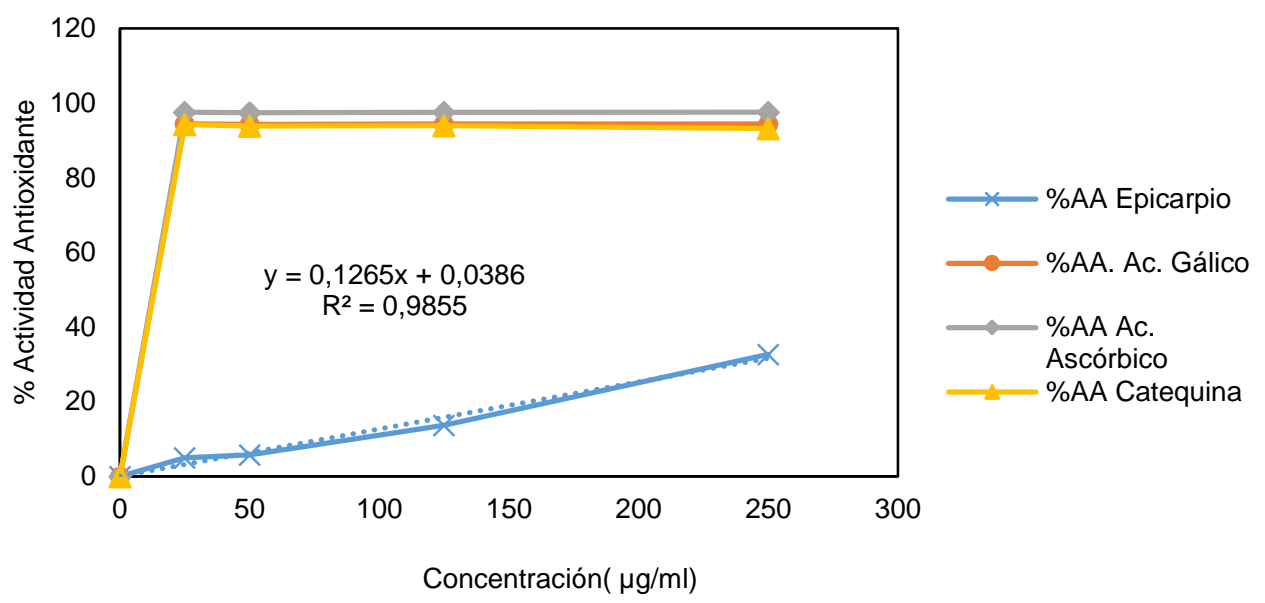

Fig. 2: Capacidad Antioxidante por DPPH.

Para el caso de EM se obtuvieron valores de $\mathrm{IC}_{50} \geq 250 \mu \mathrm{g} / \mathrm{ml}$, correspondiente a $33 \%$ de actividad antioxidante. Resultados cercanos a lo reportado por Septembre-Malaterre et al. (2016) para extractos obtenidos a partir de pulpa de mango, piña, banano (Actividad antioxidante entre 45-58\%) e inferiores a lo reportado para extracto de pulpa de maracuyá (68\% de actividad) utilizando metanol grado reactivo como solvente. La metodología ABTS es comúnmente utilizada para la determinación de actividad antioxidante en plantas, basada en la habilidad de un extracto en estabilizar el radical libre ABTS+ catión. En la figura 3 se reportan los resultados para actividad antioxidante por el método ABTS. La absorbancia correspondiente a $1.000 \mu \mathrm{M}(0.3489)$ se sustituyó en la ecuación de la recta reportada en la figura 3, con lo cual se obtuvo un valor de 2,09 $\mathrm{mm} / \mathrm{g}$ seco de EM. Valor similar a lo reportado por Martínez et al. (2012) para epicarpio de maracuyá $(2,1 \mu \mathrm{m} / \mathrm{g}$ de HEM) e inferior al comparar con extractos de piña y guayaba $(7,7$ y $20,9 \mu \mathrm{m} / \mathrm{g})$ utilizando metanol grado reactivo como solvente. La magnitud de la capacidad antioxidante depende de la matriz estudiada y su variación podría estar relacionada con la composición polifenólica del extracto. La baja actividad antioxidante en relación a otros extractos se podría atribuir a los tiempos de secado (18 horas) y tipo de solvente, ya que de acuerdo a lo reportado por Nascimento et al. (2016), los tiempos extensos y temperaturas elevadas de secado pueden desencadenar en la generación y acumulación de melanoidinas por reacciones de Maillard, a su vez la naturaleza del solvente (polar o apolar) permite tener un mayor o menor espectro de metabolitos extraídos, con lo cual se podría ver afectado el contenido de compuestos fenólicos y carotenoides, en consecuencia, la disminución de la capacidad antioxidante.

Diferentes autores han reportado el contenido de polifenoles en extracto de epicarpio de maracuyá (Chau y Huang, 2004; López-Vargas et al., 2013; Hernández y Santos et al., 2015) y la presencia de estructuras químicas de base flavonol a los cuales se les atribuye capacidad antioxidante, tales como quercetina, resveratrol y epicatequina (Septembre-Malaterre et al., 2016), dicha actividad podría prevenir la hiperglucemia en dietas controladas, según experimentos desarrollados en ratones, donde se incorporó EM en una ingesta alta en grasa y azúcar, observándose una disminución considerable de estos parámetros (Lima et al., 2016). Por otro lado, el alto contenido de fibra dietaría insoluble (44,84 g/100 g), influyó en la capacidad antioxidante de EM, gracias a la presencia de lignina clasificada en el grupo de los polifenoles y la presencia de carotenoides (3.8 mg $\beta$-carotenos $/ 100 \mathrm{~g} \mathrm{b.s.)} \mathrm{(tabla} \mathrm{1)} \mathrm{las} \mathrm{cuales} \mathrm{se} \mathrm{les} \mathrm{atribuye} \mathrm{la} \mathrm{capacidad}$ de inhibir radicales libres (Martinez et al., 2012).
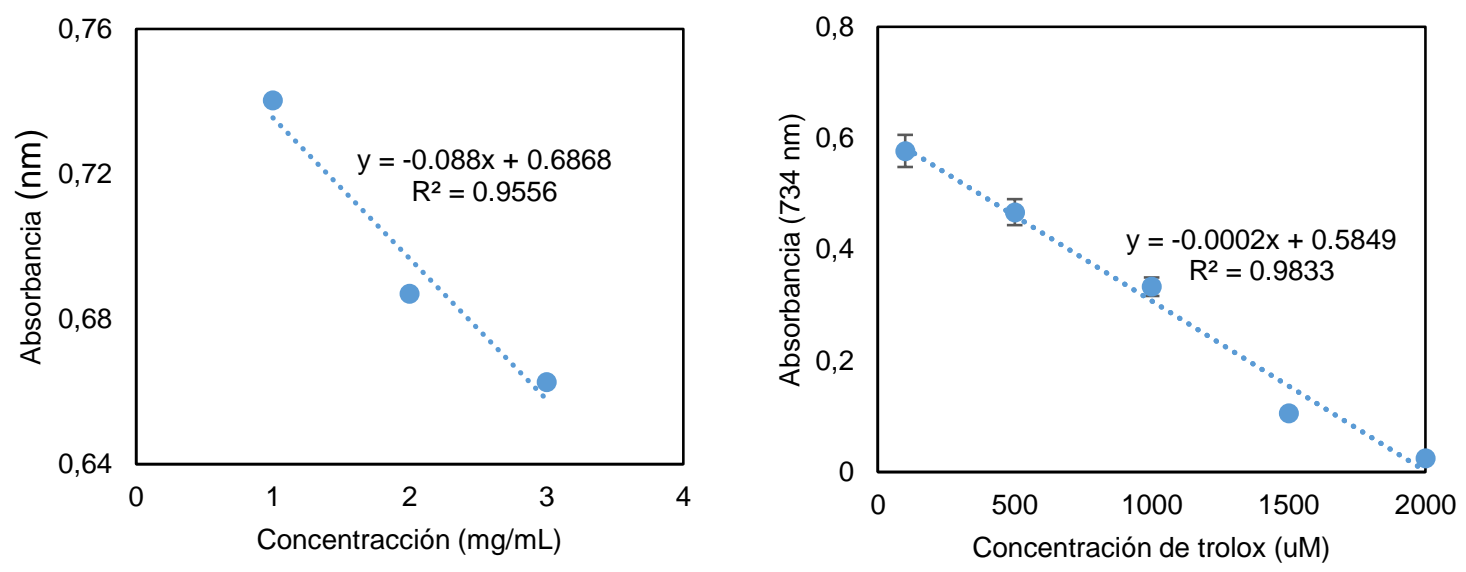

Fig.3: Método ABTS. a. Curva patrón de trolox ; b. Capacidad antioxidante de EM 


\section{Análisis microbiológico y de metales pesados en epicarpio de maracuyá (EM)}

De acuerdo a lo reportado en la tabla 3, muestras de epicarpio de maracuyá (EM) cumplieron con los requerimientos establecidos en la NTC-267 (ICONTEC,2007) para harinas vegetales. El recuento total de mesofilos (UFC/g-ml) fue de 73.000, Mohos (UFC/g) $\leq 10$, recuento de levaduras (UFC/g) 4.600, detección de Salmonella/ negativa, Recuento de Escherichia coli (UFC-g-ml) $\leq 10$, Recuento de Estafilococo Coagulasa Positiva (UFC/g-ml) $\leq 100$. Los resultados conllevan a aceptar la calidad microbiológica del epicarpio de maracuyá, por cuanto estos recuentos reflejan el cumplimiento de las Buenas Prácticas de Manufactura a lo largo del proceso de obtención, que no sólo inciden en la calidad del producto sino también en la prevención de un posible riesgo sanitario. Los resultados sobre identificación de metales pesados tambien se presentan en la tabla 3. Los niveles de plomo y cadmio identificados en el epicarpio de maracuyá estuvieron dentro de los rangos establecidos $(0,04$ y $0,01 \mathrm{mg} / \mathrm{kg})$. Al respecto Bagjuz y Hayat (2009) reportan que: En el medio agrícola, en particular en el suelo, el contenido de metales pesados debería ser únicamente función de la composición del material original. Concentraciones altas de estos metales, incluidos los esenciales para el crecimiento y desarrollo de las plantas, ejercen efectos tóxicos en sus rutas metabólicas, ya que ellos pueden bloquear grupos funcionales de moléculas importantes, sustituir iones esenciales de sitios celulares y afectar la integridad de las membranas celulares y orgánulos.

Tabla 3: Análisis microbiológico y de metales pesados ( $\mathrm{mg} / \mathrm{kg}$ ) obtenidos para epicarpio de maracuyá (EM).

\begin{tabular}{|c|c|c|c|}
\hline Análisis & Método & Especificaciones & Resultado \\
\hline $\begin{array}{l}\text { Recuento total de Aerobios } \\
\text { Mésofilos (UFC/g-ml) }\end{array}$ & NTC 4519 & 300,000 Max. & 73,000 \\
\hline Recuento de mohos (UFC/g) & NTC 5698 & 5,000 Max & $\leq 10$ \\
\hline Recuento de Levaduras (UFC/g) & NTC 5698 & 5,000 Max & 4,600 \\
\hline $\begin{array}{l}\text { Recuento de Escherichia coli } \\
\text { (UFC-g-ml) }\end{array}$ & NTC 4458 & $\leq 10$ & $\leq 10$ \\
\hline Salmonella en $25 \mathrm{~g}$ & NTC 4574 & Ausencia & Ausencia \\
\hline $\begin{array}{l}\text { Recuento de Estafilococo } \\
\text { Coagulasa Positiva (UFC/g-ml) }\end{array}$ & NTC 4679 & $\leq 100$ & $\leq 100$ \\
\hline \multicolumn{4}{|l|}{ Metales pesados (mg/kg) } \\
\hline Plomo & ICP-OES & 0,2 & 0,04 \\
\hline Cadmio & ICP-OES & 0,2 & 0,01 \\
\hline
\end{tabular}

\section{CONCLUSIONES}

El epicarpio de maracuyá representa un ingrediente potencial para la industria alimentaria, debido al alto contenido de fibra dietaría y el balance entre fibra soluble e insoluble, los cuales tienen en un efecto benéfico en la salud humana. De igual forma su, alta capacidad emulsionante, capacidad de hinchamiento y alta solubilidad le permite ser usado como ingrediente en alimentos funcionales con el fin de disminuir el contenido de grasa, problemas de sinéresis y modificar la textura y viscosidad del producto. Teniendo en cuenta que este residuo se dispone de manera inapropiada al medio ambiente, su uso implicaría una posible disminución en el impacto ambiental reflejado en la reducción de los niveles de DBO necesarios para el equilibrio del ecosistema. Adicionalmente su actividad antioxidante asociada al contenido de compuestos bioactivos (carotenoides y polifenoles) y los beneficios que aportan a la salud, le confieren también un potencial uso en la industria farmacéutica.

\section{AGRADECIMIENTOS}

Los autores agradecen a la Facultad de Ingeniería y Administración de la Universidad Nacional de Colombia sede Palmira, por el apoyo financiero otorgado mediante Proyecto Hermes No. 35763. 


\section{REFERENCIAS}

AGRONET., Producción en el departamento del Valle del Cauca (2013)

A.O.A.C. 991.43, Official Methods of Analysis international, agricultural chemicals, contaminants, drugs.15a Edición Maryland, EE.UU. (1990)

A.O.A.C. 942.15, Official Methods of Analysis international, agricultural chemicals, contaminants, drugs.15a Edición Maryland, EE.UU. (1990)

A.O.A.C. 981.12, Official Methods of Analysis international, agricultural chemicals, contaminants, drugs.15a Edición Maryland, EE.UU. (1990)

A.O.A.C. 925.09, Official Methods of Analysis international, agricultural chemicals, contaminants, drugs. 17ª Edición Maryland, EE.UU. (2000)

A.O.A.C. 978.19, Official Methods of Analysis international, agricultural chemicals, contaminants, drugs. 17ª Edición Maryland, EE.UU. (2000)

A.O.A.C. 923.03, Official Methods of Analysis international, agricultural chemicals, contaminants, drugs. 18ª Edición Maryland, EE.UU. (2005)

Bagjuz, A. y S. Hayat, Effects of brassinosteroids on the plant responses to environmental stresses, doi: 10.1016/j.plaphy.2008.10.002, Plant Physiol. Biochem., 47(1), 1-8 (2009)

Bligh, E.G. y W.J. Dyer, A rapid method of total lipid extraction and purification, Canadian Journal of Biochemistry and Physiology, 37, 911-917 (1959)

Chau, C.F. e Y.L. Huang, Characterization of passion fruit seed fibres - a potential fibre source, doi: 10.1016/j.foodchem.2003.05.009, Food Chem., 85(2), 189-194 (2004)

Crizel, T.M., A. Jablonski y otros tres autores, Dietary fiber from orange by products as a potential fat replacer, doi: 10.1016/j.Iwt.2013.02.002, Food Sci. and Tech., 53 (1), 9-14 (2013)

Da Costa, J.N., R. W. Figueiredo y otros cuatro autores, Study of the stability of passion fruit (Passiflora edullis $f$. flavicarpa) powder from organic farming, doi:10.5433/1679-0359.2013v34n2p705, Semina: Ciencias Agrarias., 34(2), 705-716 (2013)

Elleuch, M., D. Bedigian y otros cuatro autores, Dietary fibre and fibre-rich by-products of food processing: Characterisation, technological, functionality and commercial applications: A review, doi: 10.1016/j.foodchem.2010.06.077, Food Chem., 124(2), 411- 421 (2011)

Espirito Santo, A. P., P. Perego y A.M.N. Converti, Influence of milk type and addition of passion fruit peel powder on fermentation kinetics, texture profile and bacterial viability in probiotic yogurts, doi:10.1016/j.Iwt.2012.01.038, LWT- Food Sci. and Technol., 42(2), 393-399 (2012)

FAO, Tropical fruit. (2014)

Fernandez- Lopez, J., E. Sendra y otros tres autores, Physico-chemical and microbiological profiles of salchichón (Sapnish dry-fermented sausage) enriched with orange fiber, doi: 10.1016/j.meatsci.2008.01.010, Meat Sci., 80(2), 410417 (2008)

Hernández - Santos, B., M. A. Vivar- Vera y otros cinco autores, Dietary fibre and antioxidant compounds in passion fruit (Passion edulis f), doi:10.1111/ijfs.12647, Int. J. of Food Sci. and Technol., 50(1), 268-274 (2015)

Kaur, M. y N. Singh, Studies on functional, thermal and pasting properties of flours from different chickpea (Cicer arietinum L.) cultivars, doi: 10.1016/j.foodchem.2004.06.015, Food Chem., 91(3), 403-411 (2005)

Larrauri, J.A., P. Ruperez y F. Saura-Calixto, Effect of drying temperature on the stabilitity of polyphenols and antioxidant activity of red grape pomace peels, doi:10.1021/jf960282f, J. of Agr. and Food Chem., 45(4), 1390-1393 (1997)

Larrauri, J.A., New approaches in the preparation of high dietary fibre powders form fruit by-products, doi:10.1016/s09242244(99)00016-3, Trends in food Sci. \& Technol., 10(1), 3-8 (1999)

Lima, G.C., M.M. Vuolo y otros cuatro autores, Passiflora edulis peel intake improves insulin sensitivity, increasing incretins and hypothalamic satietogenic neuropeptide in rats on a high-fat diet, doi: 10.1016/j.nut.2016.01.014, Nutrition, 32(7-8), 863-870 (2016)

Lopez- Vargas, J.H., J. Fernandez- Lopez y otros dos autores, Chemical, physico-chemical, technological, antibacterial and antioxidant properties of dietary fiber powder obtained from yellow passion fruit (Passifloraedulis var.flavicarpa) coproducts, doi:10.1016/j.foodres.2013.01.055, Food Res. Int., 51(2), 756-763 (2013)

Lopez- Vargas, J.H., J. Fernandez- Lopez y otros dos autores, Quality characteristics of pork burger added with albedofiber powder obtained from yellow passion fruit (Passifloraedulisvar. flavicarpa) co-products, doi: 10.1016/j.meatsci.2014.02.010, Meat Sci., 97(2), 270-276 (2014)

Macagnan, T.F., R. L. Do Santos y otros tres autores, Biological properties of apple pomace, orange bagasse and passion fruit peel as alternative sources of dietary, doi: 10.1016/j.bcdf.2015.04.001, Bioact. Carb. and Dietary Fibre., $6(1), 1-6(2015)$ 
Macagnan, T.F., P.L. Da Silva y L.H. Hecktheuer, Dietary fiber: The scientific search for an ideal definition and methodology of analysis,and its physiological importance as a carrier of bioactive compunds, doi:10.1016/j.foodres.2016.04.032, Food Reaearch Int., 85(-), 144-154 (2016)

Marques, F. S. S., F. M. R. Libonati y otros seis autores, Evaluation of the effects of passion fruit peel flour (Passiflora edulisvar. Flavicarpa) on metabolic changes in HIV patients with lipodystrophy siyndrome secondary to antiretroviral therapy, doi: 10.1016/j.bjp.2016.03.002, Brazilian J. of Pharmacognosy., 26(4), 420-426 (2016)

Martínez-Cervera, S., A. Salvador y otros tres autores, Cocoa fibre and its application as a fat replacer in chocolate muffins, doi: 10.1016/j.Iwt.2010.06.035, LWT - Food Sci. and Tech., 44(3), 729-736 (2011)

Martínez, R., P. Torres, M. A. Meneses y otros tres autores, Chemical, technological and in vitro antioxidant properties of mango, guava, pineapple and passion fruit dietary fibre concentrate, doi: 10.1016/j.foodchem.2012.05.057, Food Chem., 135(3), 1520-1526 (2012)

Mensor, L. L., F. Menezes y otros cinco autores, Screening of Brazilian Plant Extracts for Antioxidant Activity by the Use of DPPH Free Radical Method, doi:10.1002/ptr.687, Phytotherapy research phytother., 15(2), 127-130 (2001)

Mudgil, D. y S. Barak, Composition, properties and health benefits of in-digestible carbohydrate polymers as dietary fiber: Areview, doi: 10.1016/j.ijbiomac.2013.06.044, Int. J. of Biological Macromolecules, 61, 1-6 (2013)

Nascimento, E.M. G.C., A. Mulet y otros tres autores, Effects of high-intensity ultrasound on drying kinetics and antioxidant properties of passion fruit peel, doi: 10.1016/j.jfoodeng.2015.09.015, J. of Food Eng. 170, 108-118 (2016)

NTC. 267, Instituto Colombiano de Normas Técnicas y Certificación, Harina de trigo. ICONTEC, Colombia-Bogotá (2007)

Nenandis, N., L. F. Wang y otros dos autores, Estimation of scavenging activity of phenolic compounds ising the ABTS-+ assay, doi:10.1021/jf0400056, J. of Agr. and Food Chem., 52(15), 4669-4674 (2004)

Ordoñez - Santos, L.E., P. Hurtado y otros dos autores, Concentración de carotenoides totales en residuos de frutas tropicales, doi:10.22507/pml. v9n1a7, Producción + Limpia, 9(1), 91-98 (2014)

Sánchez-Zapata, E., J. Fernández-López y otros cinco autores, Technological properties of date paste obtained from date by-products and its effect on the quality of a cooked meat product, doi: 10.1016/j.foodres.2010.04.034, Food Research Int., 44(7), 2401-2407 (2011)

Scott-Thomas, C., How the 2010 guidelines affect food technologists (2011)

Septembre - Malaterre, Stanislas, G., E. Douraguia y M-P. Gonthier, Evaluation of nutritional and antioxidant properties of the tropical fruits banana, litchi, mango, papaya, passion fruit and pineapple cultivated in Réunion French Island, doi: 10.1016/j.foodchem.2016.05.147, Food Chem., 212, 225-233 (2016)

Talcott, S.T., S. S. Percival y otros dos autores, Phytochemical composition and antioxidant stability of fortifiel yellow passion fruit (Passifloraedulis), doi:10.1021/jf020769q, J. of Agr. and Food Chem., 51(4), 935-941 (2003)

Wani, I. A., D. S. Sogi y otros dos autores, Physico-chemical and functional properties of flours from Indian kidney bean (Phaseolus vulgaris L.), doi: 10.1016/j.Iwt.2013.02.006, Food Sci. and Tech., 53(1), 278-284 (2013)

Yu S., M. Ying y otros dos autores, 'Physicochemical Properties of Starch and Flour from Different Rice Cultivars', doi:10.1007/s11947-010-0330-8, Food and Biop. Tech., 5(2), 626-637 (2012) 